\title{
EFECTO DE DIETAS ENRIQUECIDAS CON VITAMINA E Y SELENIO ORGÁNICO EN EL COMPORTAMIENTO PRODUCTIVO Y CALIDAD FUNCIONAL DEL FILETE DE TRUCHAARCO IRIS (Oncorhynchus mykiss)
}

\section{Effect of Diets Enriched with Vitamin E and Organic Selenium on the Productive Performance and Meat Quality of Rainbow Trout (Oncorhynchus mykiss)}

\author{
Héctor Rodríguez P. ${ }^{1,3,4}$, Sergio Rojas M..$^{2,5}$
}

\section{Resumen}

\begin{abstract}
El objetivo del presente estudio fue evaluar dietas enriquecidas con vitamina $\mathrm{E}$ y selenio orgánico sobre el comportamiento productivo y la calidad funcional del filete de truchas arco iris (Oncorhynchus mykiss). El estudio se hizo en el Centro Piscícola «El Ingenio» en Huancayo, Perú, con 4200 truchas juveniles (164 g de peso) durante 40 días. Se trabajó con una dieta basal (tratamiento 1), dos niveles de vitamina E (25 y 50 ppm y tres niveles de selenio orgánico $(0.15,0.25$ y $0.35 \mathrm{ppm})$. Se asignaron 600 peces por cada uno de los siete tratamientos y se tuvo tres repeticiones por cada uno $(\mathrm{n}=200)$ en un diseño experimental completamente al azar. Se evaluó la ganancia de peso, conversión alimenticia y eficiencia proteica. Además, se determinó el contenido de vitamina $\mathrm{E}$, selenio, nitrógeno de bases volátiles totales y el estado de oxidación en el filete congelado. Los datos se sometieron al análisis de varianza, la prueba múltiple de Tukey y la prueba de Dunnett. La suplementación de la dieta con 50 ppm de vitamina E y 0.35 ppm de selenio orgánico mejoró la ganancia de peso, la conversión alimenticia y la eficiencia proteica $(\mathrm{p}<0.05)$; asimismo, esta dieta mejoró la calidad funcional del filete de trucha en relación al grupo control $(\mathrm{p}<0.05)$, al tener la capacidad de almacenar $0.23 \mathrm{mg}$ de selenio $/ \mathrm{kg}$ de filete.
\end{abstract}

Palabras clave: trucha arco iris, selenio orgánico, vitamina E, suplementación, dietas

\footnotetext{
${ }^{1}$ Escuela de Post Grado, ${ }^{2}$ Profesor Emérito, Universidad Nacional Agraria La Molina, Lima, Perú

${ }^{3}$ Dirección actual: Universidad Nacional Jorge Basadre Grohmann, Tacna, Perú

${ }^{4}$ E-mail: hectorodriguezp@yahoo.es

${ }^{5}$ E-mail: srojasm1@yahoo.es, srojasm@lamolina.edu.pe
} 
The aim of this study was to evaluate diets enriched with vitamin $\mathrm{E}$ and organic selenium on the productive performance and meat quality of rainbow trout (Oncorhynchus mykiss). The study was carried out in the fish farm «El Ingenio» in Huancayo, Peru with 4200 juvenile trout (164 g of body weight) during 40 days. A basal diet (treatment 1$)$, two levels of vitamin E (25 and $50 \mathrm{ppm}$ ) and three levels of organic selenium $(0.15,0.25$ and $0.35 \mathrm{ppm}$ ) were used. In the study, 600 trout were assigned per each of the seven treatments, using three repetitions per treatment $(n=200)$ in a completely randomized design. Body weight gain, feed conversion ratio, and protein efficiency ratio were evaluated. Besides, in the frozen meat content of vitamin E, selenium and total volatile basic nitrogen, and the oxidation state were determined. Data was processed by the analysis of variance, the Tukey multiple comparison test and the Dunnett's test. The diet supplemented with 500 ppm of vitamin $E$ and 0.35 ppm of organic selenium improved body weight gain, feed conversion ratio and protein efficiency ratio $(p<0.05)$. Likewise, this diet improved the meat quality in relation to the control group $(\mathrm{p}<0.05)$ due to its capacity to store $0.23 \mathrm{mg}$ of selenium per kilogram of trout meat.

Key words: rainbow trout, organic selenium, vitamin E, supplementation, diets

\section{INTRODUCCIÓN}

Los suplementos nutricionales como vitaminas y minerales traza para animales de granja y peces son esenciales para su salud, bienestar y niveles productivos (Fisher, 2008). Además, la suplementación de micronutrientes mejora la calidad funcional de la carne (color, sabor, pérdida por goteo, ternura y vida útil del músculo) (Soetan et al., 2010). En la actualidad se viene empleando el selenio orgánico como parte del suplemento mineral en el alimento de animales, incluso en peces. En la producción de este nutriente, también llamado selenolevadura, se utilizan cepas de Saccharomyces cerevisiae, las cuales biotransforman el selenio inorgánico mayormente a selenometionina, que es absorbido y metabolizado como la metionina, pero con la ventaja de su mayor digestibilidad y biodisponibilidad; obviando, además, el problema del envenenamiento por selenio (Schrauzer, 2006).

Se dispone de diversos estudios en truchas arco iris (Oncorhynchus mykiss). Hilton et al. (1982) demostraron que la ab- sorción gástrica de selenio es eficiente y se le encuentra en mayor concentración en el hígado y riñón. Palace et al. (2004) indican que la selenometionina es la forma predominante de selenio, no genera radicales oxidativos y mejora el crecimiento. Küçükbay et al. (2009) determinaron que la selenometionina en truchas en condiciones de hacinamiento es más efectiva que el selenito de sodio en reducir pérdidas por los efectos perjudiciales del estrés oxidativo. Según Rider et al. (2010), la selenolevadura se absorbe y asimila en mayor medida que el selenito de sodio, aunque para Ciardullo et al. (2008), el selenio se acumula en mayor cantidad en el hígado y gónadas, y en menor proporción en el músculo.

La vitamina E se utiliza en nutrición animal y en la industria de alimentos por su función antioxidante, dado que evita la reacción de los radicales libres con moléculas como los ácidos grasos de las membranas celulares y grasas que circulan por la sangre. Solo los vegetales la pueden sintetizar, de allí que debe ser obtenida a través de los alimentos (Sen et al., 2006). 
La vitamina $\mathrm{E}$ y el selenio también actúan en forma sinérgica. La vitamina E como antioxidante liposoluble y el selenio como componente de la enzima glutatión peroxidasa (Zu e Ip, 2003). Bell et al. (1985) determinaron que la deficiencia de estos micronutrientes en truchas ocasiona diátesis exudativa y pérdida de peso. Según Oldfield (2003), la vitamina $\mathrm{E}$ y el selenio, además de proveer nutrientes, disminuyen el riesgo de padecer alguna patología al controlar el exceso de radicales libres y las acciones destructoras de las células que originan infecciones.

El objetivo de la presente investigación fue evaluar el comportamiento productivo de la trucha arco iris (O. mykiss) y la calidad funcional del filete mediante el enriquecimiento de las dietas con dos concentraciones de vitamina $\mathrm{E}$ y tres niveles de selenio orgánico.

\section{Materiales y Métodos}

\section{Lugar de Estudio y Animales}

El estudio se realizó en el Centro Piscícola «El Ingenio», ubicado en el distrito de Ingenio, provincia de Huancayo (Región Junín), Perú, a 3452 msnm. Se emplearon 4200 truchas juveniles arco iris, con peso de $164 \pm 2 \mathrm{~g}$, distribuidas en forma aleatoria en siete estanques de concreto de $9.75 \mathrm{~m}$ de largo, $2 \mathrm{~m}$ de ancho y $1.20 \mathrm{~m}$ de profundidad.

El recurso hídrico provenía del río Chía, cuyo origen son los manantiales de las zonas más altas. Las aguas contenían $0.3 \mu \mathrm{g}$ selenio/L (Laboratorio CETOX, Método de Espectrofotometría de Absorción Atómica con horno de grafito) y las características físico-químicas fueron: temperatura, de 9.5 a $11.5^{\circ} \mathrm{C}$; $\mathrm{O}_{2}$ disuelto, 7.0 a $7.5 \mathrm{ppm} ; \mathrm{pH}, 7.5$ a 8.0; alcalinidad total $\left(\mathrm{CaCO}_{3}\right), 140$ a 171; caudal, 1.20 a $1.30 \mathrm{~L} / \mathrm{s}$ por estanque; transparencia, total $(0.55$ a $0.60 \mathrm{~m})$; incoloro. El tratamiento profiláctico (limpiado y encalado) de los estanques se hizo en los 7 días previos. Cada poza para 600 peces se dividió temporalmente con bastidores de madera y malla anchovetera en tres secciones de $3.25 \times 2.0$ x $1.20 \mathrm{~m}$ (200 truchas juveniles por sección). El periodo experimental de alimentación duró 40 días, entre noviembre y diciembre de 2009, hasta obtener el peso y tamaño comercial de 240 a $260 \mathrm{~g}$.

Para el registro del peso de los peces, primeramente se registró el peso de una tina con agua. Luego se introdujo un determinado número de truchas juveniles de una de las pozas y se realizó un nuevo registro de peso. La diferencia de los dos pesos dividido entre el número de peces corresponde al peso de cada trucha. Se utilizó una balanza mecánica 'tipo reloj' marca Kambor, calibrada en kilogramos hasta $100 \mathrm{~kg}$, con precisión de $100 \mathrm{~g}$.

\section{Dietas Experimentales y Tratamientos}

Se trabajó con seis dietas experimentales y una dieta basal, a fin de evaluar dos niveles de vitamina $\mathrm{E}$ ( 25 y $50 \mathrm{mg} / \mathrm{kg}$ dieta) y tres niveles de selenio orgánico $(0.15,0.25 \mathrm{y}$ $0.35 \mathrm{mg} / \mathrm{kg}$ de dieta). Los tratamientos fueron: $\mathrm{T}_{1}$ : Dieta basal (DB); $\mathrm{T}_{2}, \mathrm{~T}_{3 \mathrm{y}} \mathrm{T}_{4}$ : $\mathrm{DB}$ con $25 \mathrm{mg}$ vitamina $\mathrm{E} / \mathrm{kg}$ dieta $+0.15,0.35$ y 0.50 $\mathrm{mg} \mathrm{Se} / \mathrm{kg}$ de dieta, respectivamente; $\mathrm{y}_{5}, \mathrm{~T}_{6}$ $\mathrm{y}_{7}$ : con $50 \mathrm{mg}$ vitamina $\mathrm{E} / \mathrm{kg}$ dieta +0.15 , 0.25 y $0.35 \mathrm{mg} \mathrm{Se} / \mathrm{kg}$ de dieta, respectivamente. El alimento consumido por las truchas antes del experimento fue una dieta comercial extruida con $40 \%$ de proteína.

Se utilizó un diseño completamente aleatorizado, con siete tratamientos y tres repeticiones por tratamiento. Cada tratamiento tuvo 600 truchas, con los siguientes errores estándar: $\mathrm{T}_{1} \pm 0.03, \mathrm{~T}_{2} \pm 0.04, \mathrm{~T}_{3} \pm 0.05$, $\mathrm{T}_{4} \pm 0.03, \mathrm{~T}_{5} \pm 0.0_{7}, \mathrm{~T}_{6} \pm 0.05 \mathrm{y} \mathrm{T}_{7} \pm 0.02, \mathrm{y}$ cada repetición se hizo con 200 truchas.

El modelo aditivo lineal fue el siguiente: $Y i j=$ $\mu+\tau \mathrm{i}+\varepsilon \mathrm{ij}$, donde:

Yij = Es la observación con el i-ésimo tratamiento en la j-ésima repetición

$\mu=$ Efecto de la media general 
Cuadro 1. Composición de la dieta basal (DB) de truchas arco iris

\begin{tabular}{lr}
\hline Ingredientes & \multicolumn{1}{c}{$\%$} \\
\hline Harina de pescado (67\% & 31.861 \\
proteína) & 30.0 \\
Torta de soya (47\% proteína) & 20.0 \\
Harinilla de trigo & 15.0 \\
Harina de trigo & 2.0 \\
Aceite vegetal (soya) & 0.819 \\
Sal común & 0.30 \\
Premezcla de vitaminas y & 0.02 \\
minerales & 100.0 \\
\hline Antioxidante (BHT)
\end{tabular}

$\tau \mathrm{i}=$ Efecto del i-ésimo tratamiento

$\varepsilon i j=$ Efecto del error experimental en el iésimo tratamiento y en la j-ésima repetición

La dieta basal (DB) y las dietas experimentales en forma de pellets fueron elaboradas en la Planta de Alimentos Balanceados de la Universidad Nacional Agraria La Molina. El nivel de proteína fue de $38.5 \%$ y de lípidos de $15.0 \%$. En la formulación, la premezcla comercial Rovimix ${ }^{\circledR}$, premix para acuicultura (Cuadro 1) contenía además: 140000 UI de $\alpha$-tocoferol y $0.3 \mathrm{~g}$ de selenito de sodio, ambos para una tonelada de alimento. Las dietas fueron pesadas con una balanza electrónica digital marca Kinlee, modelo EPSO5, con capacidad de $500 \mathrm{~g}$ y precisión de $0.1 \mathrm{~g}$. La composición de la DB se presenta en el Cuadro 1.

Se utilizó la vitamina E comercial, que contiene $50 \%$ de DL- $\alpha$-tocoferol, y como selenio orgánico a Sel-Plex (insumo procedente de la biotransformación del selenio inorgánico a selenometionina, selenocistina y selenocisteína por la levadura $S$. cerevisiae).

\section{Alimentación}

El manejo y alimentación de las truchas fue bajo las mismas condiciones de la piscigranja. La distribución del alimento fue en forma manual, al voleo. Se repartió por la mañana y por la tarde.

La cantidad de alimento fue determinada mediante la Tasa de Alimentación de 1.6\% (porcentaje de la biomasa de las truchas presentes), recomendado por la Planta de Alimentos Balanceados de la UNALM.

\section{Comportamiento Productivo}

El comportamiento productivo se estimó en base a los siguientes parámetros:

- Ganancia de peso (GP). Diferencia entre peso final y peso inicial (g), sobre los 40 días del experimento (Hopkins, 1992).

- Conversión alimenticia (CA). Definido por el consumo de alimento en 40 días sobre la ganancia de peso en ese mismo periodo (NTP, 2008).

- Relación de Eficiencia Proteica (PER). Gramos de peso ganado por gramo de proteína consumida.

- Mortalidad (método del conteo directo). Se llevó un registro diario de mortalidad.

\section{Calidad Funcional del Filete de Trucha}

Las muestras estuvieron en congelamiento por un mes. Se hicieron las siguientes determinaciones:

- Análisis de vitamina E. Se utilizó el método de cromatografía líquida de alta resolución (HPLC). El análisis se hizo en los Laboratorios de Certificaciones del Perú (CERPER).

- Análisis de selenio total. Se hizo en CERPER, por el método de espectrofotometría de absorción atómica con generador de hidruros, descrito por la AOAC (Método Oficial 986.15, 2005).

- Análisis de nitrógeno de bases volátiles totales $(N-B V T)$. El N-BVT mide el grado de frescura de la trucha. El análisis 
Cuadro 2. Ganancia de peso (kg/día) en truchas arco iris suplementadas con vitamina E y selenio orgánico durante 40 días $^{1}$

\begin{tabular}{|c|c|c|c|c|c|}
\hline \multirow{2}{*}{$\begin{array}{l}\text { Vitamina } \mathrm{E} \\
\text { (mg/kg de dieta) }\end{array}$} & \multicolumn{4}{|c|}{ Selenio orgánico por kg de dieta } & \multirow{2}{*}{ Total } \\
\hline & $0 \mathrm{mg}$ & $0.15 \mathrm{mg}$ & $0.25 \mathrm{mg}$ & $0.35 \mathrm{mg}$ & \\
\hline 0 & $11.61\left(\mathrm{~T}_{1}\right)^{\mathrm{b}}$ & & & & 11.61 \\
\hline 25 & & $9.79\left(\mathrm{~T}_{2}\right)$ & $13.47\left(\mathrm{~T}_{3}\right)$ & $13.33\left(\mathrm{~T}_{4}\right)$ & 12.20 \\
\hline 50 & & $11.59\left(\mathrm{~T}_{5}\right)$ & $14.82\left(\mathrm{~T}_{6}\right)$ & $17.57\left(\mathrm{~T}_{7}\right)^{\mathrm{a}}$ & 14.66 \\
\hline Total & 11.61 & 10.69 & 14.15 & 15.45 & \\
\hline
\end{tabular}

se hizo en el Instituto Tecnológico de la Producción (ITP), usando el método recomendado por Directiva 95/149 de la Comunidad Económica Europea.

- Análisis del estado de oxidación. Se determinó en el ITP, mediante la cuantificación del ácido tiobarbitúrico (TBA), expresado en miligramos de malondialdehido (MDA) por kilogramo de trucha.

\section{Análisis Estadístico}

La ganancia de peso, estado de oxidación y el metabolismo del selenio orgánico y la vitamina $E$ en el cuerpo de la trucha fueron evaluados mediante un análisis de varianza. La prueba múltiple de Tukey se usó para identificar el mejor tratamiento y la prueba de Dunnett para comparar diferencias entre medias de los seis tratamientos enriquecidos contra la DB $\left(\mathrm{T}_{1}\right)$. El análisis estadístico se hizo con el software SAS (SAS, 1995), utilizando una probabilidad de $\mathrm{p}<0.05$.

\section{Resultados y Discusión}

\section{Ganancia de Peso}

La biomasa inicial y final fue de 687.27 \pm 7.14 y $965.91 \pm 27.86 \mathrm{~kg}$, respectivamente, habiendo una ganancia de peso total en 40 días de $278.64 \pm 4.85 \mathrm{~kg}(40.5 \%)$. Asimismo, la carga inicial fue de $4.58 \pm 0.07 \mathrm{~kg} / \mathrm{m}^{3}$ y la carga final de $6.43 \pm 0.51 \mathrm{~kg} / \mathrm{m}^{3}$.

Los resultados de ganancia de peso se presentan en el Cuadro 2. Las truchas del $\mathrm{T}_{7}$ lograron una ganancia de peso promedio de $17.57 \pm 1.39 \mathrm{~kg} / \mathrm{d}$ ía para el grupo de 200 peces, en comparación con las truchas del grupo control $\left(\mathrm{T}_{1}\right)$ con $11.61 \pm 1.06 \mathrm{~kg} /$ día $(\mathrm{p}<0.05)$, lo cual indica un incremento de $51 \%$. Estos datos no han podido ser comparados con resultados de otros trabajos, dado que se desconocen estudios sobre ganancia de peso en truchas $O$. mykiss por efecto de la vitamina $\mathrm{E}$ y el selenio orgánico.

El análisis de varianza indicó que hubo diferencias estadísticas entre tratamientos, en tanto que las pruebas de Tukey y Dunnett mostraron diferencias estadísticas $(p<0.05)$ por efecto de la vitamina $\mathrm{E}$ y el selenio orgánico. Es decir, los peces con la dieta suplementada con $50 \mathrm{mg}$ de vitamina E y $0.35 \mathrm{mg}$ de selenio $\mathrm{T}_{7}$ ganaron más peso diario $(2.20$ $\pm 0.07 \mathrm{~g} /$ día $)$ que los del control $\mathrm{T}_{1}(1.45 \pm$ $0.17 \mathrm{~g} /$ día). Esta diferencia demostraría el efecto de sinergia de la vitamina $E$ y el selenio orgánico. En cambio, la no significativa aunque aparente mayor ganancia de peso de truchas del $\mathrm{T}_{1}$ sobre $\mathrm{T}_{2}(1.23 \pm 0.19 \mathrm{~g} / \mathrm{d}) \mathrm{y} \mathrm{T}_{5}$ 
Cuadro 3. Conversión alimenticia (CA) en truchas arco iris suplementadas con vitamina E y selenio orgánico durante 40 días $^{1}$

\begin{tabular}{cccccc}
\hline \multirow{2}{*}{$\begin{array}{l}\text { Vitamina E } \\
(\mathrm{mg} / \mathrm{kg} \text { de dieta) }\end{array}$} & \multicolumn{3}{c}{ Selenio orgánico por kg de dieta } & \multirow{2}{*}{ Total } \\
\cline { 2 - 4 } & $0 \mathrm{mg}$ & $0.15 \mathrm{mg}$ & $0.25 \mathrm{mg}$ & $0.35 \mathrm{mg}$ & \\
\hline 0 & $1.84\left(\mathrm{~T}_{1}\right)^{\mathrm{b}}$ & & & 1.84 \\
25 & & $2.16\left(\mathrm{~T}_{2}\right)$ & $1.64\left(\mathrm{~T}_{3}\right)$ & $1.59\left(\mathrm{~T}_{4}\right)$ & 1.80 \\
50 & & $1.83\left(\mathrm{~T}_{5}\right)$ & $1.44\left(\mathrm{~T}_{6}\right)$ & $1.20\left(\mathrm{~T}_{7}\right)^{\mathrm{a}}$ & 1.49 \\
\hline Total & 1.84 & 2.00 & 1.54 & 1.40 \\
\hline 1 Cada grupo de 200 truchas consumió 21.12 kg de alimento \\
a La prueba múltiple de Tukey mostró diferencia significativa $(\mathrm{p}<0.05)$ entre tratamientos a favor de $\mathrm{T}_{7}$ \\
b La prueba múltiple de Dunnett reveló que solo la diferencia $\left(\mathrm{T}_{7}-\mathrm{T}_{1}\right)$ es significativa $(\mathrm{p}<0.05)$
\end{tabular}

$(1.45 \pm 0.15 \mathrm{~g} / \mathrm{d})$, estos últimos suplementados con $0.15 \mathrm{mg}$ de selenio orgánico, indicaría que para darse el efecto de sinergia se requeriría suplementar un mayor nivel de selenio orgánico.

\section{Conversión Alimenticia}

En el Cuadro 3 se muestran los niveles de CA por tratamiento. Las truchas del $\mathrm{T}_{7}$ requirieron consumir $1.20 \pm 0.08 \mathrm{~kg}$ de alimento para aumentar $1 \mathrm{~kg}$ de peso, en tanto que las truchas del grupo control $\left(\mathrm{T}_{1}\right)$ necesitaron $1.84 \pm 0.28 \mathrm{~kg}$ de alimento ( $\mathrm{p}<0.05)$, lo cual significa un ahorro del $35 \%$ de alimento por kilogramo de incremento de peso a favor del $\mathrm{T}_{7}$. Por otro lado, no hubo diferencias estadísticas en los demás tratamientos.

Morales (2004), criando truchas en 'jaulas' determinó una CA de 1.13 para una tasa de alimentación de $2.19 \%$ y de 1.32 para una tasa de alimentación de $4.41 \%$, concluyendo que la CA se maximiza con una mayor tasa de alimentación. En el presente estudio se obtuvo una CA levemente mayor y con una tasa de alimentación menor, generando menor gasto por dieta. Montaña et al. (2013), alimentando truchas 3 veces/día, logró una CA eficiente de 1.17 y 1.43 . Por otro lado, Sarmiento (2011), encontró que a mayor densidad de carga animal $\left(10,20\right.$ y $\left.30 \mathrm{~kg} / \mathrm{m}^{3}\right)$ es mayor la CA (1.91, 2.02 y 2.15, respectivamente), siendo antieconómico debido al estrés que genera el exceso de población de truchas. En el presente estudio se optó una carga de 4.15 al inicio y de $6.43 \mathrm{~kg} / \mathrm{m}^{3}$ al final, siguiendo el patrón de carga del Centro Piscícola, el cual resultó ser adecuado.

\section{Relación de Eficiencia Proteica}

Los resultados de PER se muestran en el Cuadro 4. Las truchas del $\mathrm{T}_{7}$ obtuvieron un PER promedio de $2.16 \pm 0.13$, en tanto que fue de $1.41 \pm 0.20$ en las truchas del grupo control $\left(\mathrm{T}_{1}\right)(\mathrm{p}<0.05)$; esto es, $52 \%$ superior. El valor 2.16 es aceptable, porque se acerca al PER de la caseína (2.5), el cual se toma como referencia (Allison, 1955, citado por Morris et al. 2003). Por lo tanto, la presencia de ambos nutrientes antioxidantes, sobre todo, en $\mathrm{T}_{7}$, mejoró la absorción y el metabolismo de los aminoácidos de la harina de pescado de la dieta, elevando la eficiencia proteica. Por otro lado, no hubo diferencias estadísticas entre los demás tratamientos.

Okumus y Mazlum (2002) reportan valores entre 2.03 y 2.08 de PER en truchas alimentadas con tres dietas por 22 semanas con un contenido de proteína cruda entre 40 a 48\%. Asimismo, Kiaalvandi et al. (2011) señalan valores de 1.87 a 2.18 de PER, en 
Cuadro 4. Índice de eficiencia proteica (PER) ${ }^{1}$ en truchas arco iris suplementadas con vitamina E y selenio orgánico durante 40 días $^{2,3}$

\begin{tabular}{|c|c|c|c|c|c|}
\hline \multirow{2}{*}{$\begin{array}{l}\text { Vitamina } \mathrm{E} \\
(\mathrm{mg} / \mathrm{kg} \text { de dieta) }\end{array}$} & \multicolumn{4}{|c|}{ Selenio orgánico por kg de dieta } & \multirow{2}{*}{ Total } \\
\hline & $0 \mathrm{mg}$ & $0.15 \mathrm{mg}$ & $0.25 \mathrm{mg}$ & $0.35 \mathrm{mg}$ & \\
\hline 0 & $1.42\left(\mathrm{~T}_{1}\right)^{\mathrm{b}}$ & & & & 1.42 \\
\hline 25 & & $1.20\left(\mathrm{~T}_{2}\right)$ & $1.65\left(\mathrm{~T}_{3}\right)$ & $1.63\left(\mathrm{~T}_{4}\right)$ & 1.49 \\
\hline 50 & & $1.42\left(\mathrm{~T}_{5}\right)$ & $1.82\left(\mathrm{~T}_{6}\right)$ & $2.16\left(\mathrm{~T}_{7}\right)^{\mathrm{a}}$ & 1.80 \\
\hline Total & 1.42 & 1.31 & 1.74 & 1.90 & \\
\hline \multicolumn{6}{|c|}{$\begin{array}{l}{ }^{1} \text { Ganancia de peso / proteína consumida } \\
{ }^{2} \text { Cada grupo de } 200 \text { truchas consumió } 21.12 \mathrm{~kg} \text { de alimento } \\
{ }^{3} \text { La dieta contenía } 38.54 \% \text { de proteína cruda y se registró un consumo de } 24.419 \mathrm{~kg} \text { de proteína por } \\
\text { tratamiento } \\
\text { a La prueba múltiple de Tukey mostró diferencia significativa }(p<0.05) \text { entre tratamientos a favor de } T_{7} \\
\text { b La prueba múltiple de Dunnett reveló que solo la diferencia }\left(T_{7}-T_{1}\right) \text { es significativa }(p<0.05)\end{array}$} \\
\hline
\end{tabular}

Cuadro 5. Contenido de vitamina $\mathrm{E}(\mathrm{mg} / \mathrm{kg})$ en truchas arco iris suplementadas con vitamina E y selenio orgánico durante 40 días

\begin{tabular}{cccccc}
\hline \multirow{2}{*}{$\begin{array}{l}\text { Vitamina E } \\
(\mathrm{mg} / \mathrm{kg} \text { de dieta) }\end{array}$} & \multicolumn{4}{c}{ Selenio orgánico por kg de dieta } & \multirow{2}{*}{ Total } \\
\cline { 2 - 5 } & $0 \mathrm{mg}$ & $0.15 \mathrm{mg}$ & $0.25 \mathrm{mg}$ & $0.35 \mathrm{mg}$ & \\
\hline 0 & $14.43\left(\mathrm{~T}_{1}\right)$ & & & & 14.43 \\
25 & & $12.71\left(\mathrm{~T}_{2}\right)$ & $10.52\left(\mathrm{~T}_{3}\right)$ & $14.70\left(\mathrm{~T}_{4}\right)$ & 12.64 \\
50 & & $18.54\left(\mathrm{~T}_{5}\right)$ & $15.19\left(\mathrm{~T}_{6}\right)$ & $12.03\left(\mathrm{~T}_{7}\right)$ & 15.25 \\
\hline Total & 14.43 & 15.63 & 12.86 & 13.37 & \\
\hline
\end{tabular}

truchas alimentadas con dietas de 22 a $40 \%$ de proteína, principalmente de harina de pescado. Contrariamente, Bórquez et al. (1999) hallaron valores de PER de 0.957 para dietas pelletizadas y de 0.720 para extruido, con 40 y $44 \%$ de proteína de harina de pescado, respectivamente, sin mencionar las razones de la baja eficiencia. Asimismo, Gümüş e Ikiz (2009) obtuvieron bajos valores de PER (0.90 a 1.42) con dietas con $40 \%$ de proteína, pero de origen vegetal, la cual tiene alta presencia de carbohidratos y no contiene todos los aminoácidos esenciales como la proteína de harina de pescado ni los nutrientes antioxidantes del presente estudio.

\section{Contenido de Vitamina $\mathbf{E}$}

El contenido de vitamina $\mathrm{E}$ en el filete de las truchas se muestra en el Cuadro 5. Se observa un amplio rango, variando entre 10.52 $\pm 0.37 \mathrm{mg} / \mathrm{kg}$ en $\mathrm{T}_{3}$ hasta $18.54 \pm 3.77 \mathrm{mg} / \mathrm{kg}$ en $\mathrm{T}_{5}$, pero sin diferencias estadísticas entre tratamientos. Es de esperarse que mayores diferencias pudieran encontrarse en la grasa por ser una vitamina liposoluble.

La vitamina $\mathrm{E}$ en raciones de truchas regula diversos procesos biológicos, mejora la respuesta inmune y protege contra radicales libres (Rowley et al., 1995; Pearce et al., 
Cuadro 6. Contenido de selenio $(\mathrm{mg} / \mathrm{kg}$ ) en tru chas arco iris suplementadas con vitamina $\mathrm{E}$ y selenio orgánico durante 40 días

\begin{tabular}{cccccc}
\hline \multirow{2}{*}{$\begin{array}{l}\text { Vitamina E } \\
(\mathrm{mg} / \mathrm{kg} \text { de dieta })\end{array}$} & \multicolumn{3}{c}{ Selenio orgánico por kg de dieta } & \multirow{2}{*}{ Total } \\
\cline { 2 - 5 } & $0 \mathrm{mg}$ & $0.15 \mathrm{mg}$ & $0.25 \mathrm{mg}$ & $0.35 \mathrm{mg}$ & \\
\hline 0 & $0.11\left(\mathrm{~T}_{1}\right)^{\mathrm{b}}$ & & & & 0.11 \\
25 & & $0.15\left(\mathrm{~T}_{2}\right)$ & $0.17\left(\mathrm{~T}_{3}\right)$ & $0.22\left(\mathrm{~T}_{4}\right)^{\mathrm{c}}$ & 0.18 \\
50 & & $0.16\left(\mathrm{~T}_{5}\right)$ & $0.17\left(\mathrm{~T}_{6}\right)$ & $0.23\left(\mathrm{~T}_{7}\right)^{\mathrm{a}}$ & 0.19 \\
\hline Total & 0.11 & 0.16 & 0.17 & 0.23 & \\
\hline a,b,c La prueba de Dunnett reveló que las diferencias $\left(\mathrm{T}_{7}-\mathrm{T}_{1}\right)$ y $\left(\mathrm{T}_{4}-\mathrm{T}_{1}\right)$ fueron significativas $(\mathrm{p}<0.05)$
\end{tabular}

Cuadro 7. Contenido de nitrógeno de bases volátiles (N-B VT) (mg/100 g) en truchas arco iris suplementadas con vitamina $\mathrm{E}$ y selen io orgánico durante 40 días

\begin{tabular}{cccccc}
\hline \multirow{2}{*}{$\begin{array}{l}\text { Vitamina E } \\
(\mathrm{mg} / \mathrm{kg} \text { de dieta) }\end{array}$} & \multicolumn{4}{c}{ Selenio orgánico por kg de dieta } & \multirow{2}{*}{ Total } \\
\cline { 2 - 5 } & $0 \mathrm{mg}$ & $0.15 \mathrm{mg}$ & $0.25 \mathrm{mg}$ & $0.35 \mathrm{mg}$ & \\
\hline 0 & $14.4\left(\mathrm{~T}_{1}\right)$ & & & & 14.4 \\
25 & & $14.8\left(\mathrm{~T}_{2}\right)$ & $11.6\left(\mathrm{~T}_{3}\right)$ & $12.4\left(\mathrm{~T}_{4}\right)$ & 12.9 \\
50 & & $11.2\left(\mathrm{~T}_{5}\right)$ & $12.9\left(\mathrm{~T}_{6}\right)$ & $13.5\left(\mathrm{~T}_{7}\right)$ & 12.5 \\
\hline Total & 14.4 & 13.0 & 12.3 & 13.0 & \\
\hline
\end{tabular}

2003). Se ha demostrado un efecto positivo en los ácidos grasos poliinsaturados (AGPI) y sobre el estrés por alta densidad (Yildiz, 2004), en tanto que Packer (1991) y Van Dam et al. (2003) sostienen que previene la peroxidación de los lípidos de las membranas celulares. Por otro lado, se ha demostrado un efecto estabilizador en los lípidos en filetes de pescado almacenados en refrigeración y en congelación (Jittinandana et al., 2006; Trushenski y Kohler, 2007).

\section{Concentración de Selenio}

El contenido de selenio en el filete se presenta en el Cuadro 6. No se observan diferencias estadísticas entre tratamientos al análisis con la prueba múltiple de Tukey, pero la diferencia entre medias de Dunnett entre $\mathrm{T}_{7}(0.23 \pm 0.01 \mathrm{mg} / \mathrm{kg})$ y $\mathrm{T}_{1}(0.11 \pm 0.03 \mathrm{mg} /$ $\mathrm{kg})$ resultó significativa $(\mathrm{p}<0.05)$.

Desde el punto de vista nutricional y de salud pública es conveniente obtener un alimento funcional en base a su contenido de selenio (Sara et al., 2010; Vinchira y MuñozRamírez, 2010). Esto podría garantizarse con la dieta que contiene $0.35 \mathrm{ppm}$ de selenio, pues se obtiene la presencia de ácidos grasos poliinsaturados (AGPI) de la familia $\omega-3$ (EPA y DHA), reconocidos por sus características funcionales en la prevención y reducción de enfermedades cardiovasculares (Araya y Lutz, 2003; Chen et al., 2006), dan- 
Cuadro 8. Contenido de ácido tiobarbitúrico (TBA) (mg MDA/kg) en truchas arco iris suplementadas con vitamina E y selenio orgánico durante 40 días

\begin{tabular}{cccccc}
\hline \multirow{2}{*}{$\begin{array}{l}\text { Vitamina E } \\
(\mathrm{mg} / \mathrm{kg} \text { de dieta })\end{array}$} & \multicolumn{3}{c}{ Selenio orgánico por kg de dieta } & \multirow{2}{*}{ Total } \\
\cline { 2 - 5 } & $0 \mathrm{mg}$ & $0.15 \mathrm{mg}$ & $0.25 \mathrm{mg}$ & $0.35 \mathrm{mg}$ & \\
\hline 0 & $1.16\left(\mathrm{~T}_{1}\right)$ & & & & 1.16 \\
25 & & $1.44\left(\mathrm{~T}_{2}\right)$ & $0.93\left(\mathrm{~T}_{3}\right)$ & $1.32\left(\mathrm{~T}_{4}\right)$ & 1.23 \\
50 & & $1.49\left(\mathrm{~T}_{5}\right)$ & $0.80\left(\mathrm{~T}_{6}\right)$ & $0.96\left(\mathrm{~T}_{7}\right)$ & 1.09 \\
\hline Total & 1.16 & 1.47 & 0.87 & 1.14 & \\
\hline
\end{tabular}

do la posibilidad de crear sistemas acuícolas para desarrollar nuevos alimentos funcionales con contenido de $\omega$-3 y de selenio orgánico.

\section{Nitrógeno de Bases Volátiles Totales (N- BVT)}

En el Cuadro 7 se presenta el contenido de N-BVT en el filete de las truchas, sin haberse encontrado diferencias significativas entre tratamientos. Se entiende por N-BVT la presencia de bases volátiles (trimetilamina, dimetilamina y $\mathrm{NH}_{3}$ ) y aminas de la descarboxilación microbiana de aminoácidos (Özogul y Özogul, 2000). El N-BVT es un indicador de la frescura de la carne.

Los valores de N-BVT fueron similares al valor de $12.42 \mathrm{mg} / 100 \mathrm{~g}$ reportado en truchas por Oğuzhan y Angiş (2012) y se encuentran dentro de los límites de frescura aceptables para pescado. Ruiz-Capillas y Horner (1999) señalan que el pescado recién capturado debe tener valores entre 10 a 15 $\mathrm{mg} / 100 \mathrm{~g}$ de muestra, a excepción de los peces pelágicos. Según Márquez et al. (2006), el nivel máximo tolerable de N-BVT, y que coincide con el inicio de la alteración organoléptica de la carne de peces grasos como la trucha, es de $30 \mathrm{mg} / 100 \mathrm{~g}$.
Cuadro 9. Mortalidad en truchas arco iris suplementadas con vitamina $\mathrm{E}$ y selenio orgánico durante 40 días ( $n=600$ truchas por tratamiento)

\begin{tabular}{cc}
\hline Tratamiento & $\begin{array}{c}\text { Mortalidad } \\
(\%)\end{array}$ \\
\hline $\mathrm{T}_{1}$ & 0.29 \\
$\mathrm{~T}_{2}$ & 0.31 \\
$\mathrm{~T}_{3}$ & 0.29 \\
$\mathrm{~T}_{4}$ & 0.31 \\
$\mathrm{~T}_{5}$ & 0.24 \\
$\mathrm{~T}_{6}$ & 0.26 \\
$\mathrm{~T}_{7}$ & 0.29 \\
\hline Total & 1.98 \\
\hline
\end{tabular}

\section{Concentración de Malondialdehído (MDA)}

Los contenidos de TBA fueron similares entre tratamientos (Cuadro 8) sin haberse hallado diferencia estadística mediante las pruebas de Tukey y Dunnett. Los valores fueron similares a los obtenidos por 
Haghparast et al. (2010) en truchas, empleando acetato y citrato de sodio como antioxidante. En forma similar, Jasour et al. (2011), alimentando truchas con adición de vitamina $\mathrm{E}$ para impedir la oxidación primaria, obtuvieron valores menores a $1 \mathrm{mg}$ de $\mathrm{MDA} / \mathrm{kg}$.

El valor más bajo en el presente estudio $(0.80 \pm 0.18 \mathrm{mg} \mathrm{MDA} / \mathrm{kg})$ en $\mathrm{T}_{6}$ no indica algún tipo de lipoperoxidación, en tanto que el valor más alto $(1.49 \pm 0.92 \mathrm{mg} \mathrm{MDA} / \mathrm{kg})$ en $\mathrm{T}_{5}$ no llegaría al inicio de peroxidación (Suárez et al., 2009). El valor de TBA que indica buena calidad del músculo de pescado es alrededor de $5 \mathrm{mg} \mathrm{MDA} / \mathrm{kg}$, y son aceptables para el consumo humano valores hasta de $8 \mathrm{mg}$ MDA $/ \mathrm{kg}$. Por lo tanto, los bajos valores de TBA revelan que la trucha no sufrió daño lipídico (peroxidación), en particular de los ácidos grasos poliinsaturados, durante el mes de almacenamiento en frío, encontrándose que los filetes tenían una buena calidad para su consumo.

Estos resultados indicarían que la presencia de la vitamina E de la premezcla (140 UI $\alpha$-tocoferol/Kg), que activa significativamente a las enzimas antioxidantes catalasa, peroxidasa y glutatión reductasa (Gulcin et al., 2005), así como el selenio inorgánico, también presente en la premezcla $(0.3 \mathrm{mg}$ selenita de sodio/kg) y en el agua del río Chía $(0.3 \mu \mathrm{g}$ selenio/L) tuvieron efecto protector en las células y el tejido muscular, y que una vez sacrificadas las truchas, estos antioxidantes inhibieron la peroxidación lipídica. Esto sería suficiente para evitar el deterioro oxidativo del filete, como se observa en algunos tratamientos con bajos valores de TBA. Sin embargo, una vez adicionado el selenio orgánico del estudio (que activa a la enzima glutatión peroxidasa), se muestra el refuerzo a la defensa antioxidante, resultando en algunos casos, niveles aún más bajos: $\mathrm{T}_{3}(0.93 \pm 0.34 \mathrm{mg} \mathrm{MDA} / \mathrm{kg}), \mathrm{T}_{6}(0.80 \pm 0.18$ $\mathrm{mg} \mathrm{MDA} / \mathrm{kg})$ y $_{7}(0.96 \pm 0.23 \mathrm{mg} \mathrm{MDA} /$ $\mathrm{kg})$.

\section{Mortalidad}

La mortalidad fue de $1.98 \%$ para una población de 4200 truchas (Cuadro 9), comparado con el $2.5 \%$ reportado para el Centro Piscícola, así como el 2.7, 4.6 y 5.0\% reportado por Pokniak et al. (1996), Akbulut et al. (2002) y Bastardo et al. (2003), respectivamente. El 90\% de las causas de mortalidad se atribuyen al picaje de la ave predadora «martín pescador» o «huaco» Nictycorax nictycorax, en tanto que el $10 \%$ restante se podría atribuir a razones endógenas, tanto de orden sanitario como de manejo.

\section{ConClusiones}

- La suplementación de la dieta con 50 ppm de vitamina E y 0.35 ppm de selenio orgánico mejoró la ganancia de peso, la conversión alimenticia y la eficiencia proteica $(\mathrm{p}<0.05)$ de truchas juveniles arco iris (Oncorhynchus mykiss).

- La suplementación de la ración con 50 ppm de vitamina $\mathrm{E}$ y 0.35 ppm de selenio orgánico mejoró la calidad funcional del filete de trucha en relación al grupo control $(\mathrm{p}<0.05)$, al tener la capacidad de almacenar $0.23 \mathrm{mg}$ de selenio $/ \mathrm{kg}$ de filete.

\section{Literatura Citada}

1. Akbulut B, Temel Ş, Aksungur N, Aksungur M. 2002. Effect of initial size on growth rate of rainbow trout, Oncorhynchus mykiss, reared in cages on the Turkish Black Sea Coast. Turk J Fish Aquat Sci 2: 133-136.

2. Araya H, Lutz M. 2003. Alimentos funcionales y saludables. Rev Chilena Nutr 30(1): 8-14.

3. Bastardo H, Sara B, Sofía B. 2003. Masculinización de la trucha arco iris, Oncorhynchus mykiss, para obtener 
descendencia todas hembras en un criadero venezolano. Zootec Trop 21(1): 27-41.

4. Bell JG, Cowey CB, Adron JW. 1985. Some effects of vitamin $\mathrm{E}$ and selenium deprivation on tissue enzyme levels and indices of tissue peroxidation in rainbow trout (Salmo gairdneri). Br J Nutrit 53: 149-157.

5. Bórquez RA, Valdebenito IN, Dantagnan HP, Bariles SJ. 1999. Rendimientos productivos de dietas extruidas y pelletizadas en cultivo intensivo de trucha arco iris, Oncorhynchus mykiss. Rev Invest Cient Tecnol Ser Cienc Mar 4: 1-3.

6. [CERPER]. Certificaciones del Perú. 2010. Determinación de la vitamina E. Método AACC 86-06. $10^{\text {th }}$ ed 2000. [Internet]. Disponible en: http:// www.cerper.com/

7. [CERPER]. Certificaciones del Perú. 2010. Determinación del selenio: Método AOAC - 986. 15, c9. $18^{\text {th }}$ ed. 2005. Multielement Method. [Internet]. Disponible en: http://www.cerper.com/

8. Chen YC, Nguyen J, Semmens K, Beamer S, Jaczynski J. 2006. Enhancement of omega-3 fatty acid content in rainbow trout (Oncorhynchus mykiss) fillets. J Food Sci 71: C383C389.

9. Ciardullo $S$, Aureli $F$, Coni $E$, Guandalini E, Iosi F, Raggi A, et al. 2008. Bioaccumulation potential of dietary arsenic, cadmium, lead, mercury, and selenium in organs and tissues of rainbow trout (Oncorhynchus mykiss) as a function of fish growth. J Agric Food Chem 56: 2442-2451.

10. Fisher GEJ. 2008. Micronutrients and animal nutrition and the link between the application of micronutrients to crops and animal health. Turk J Agric For 32: 221233.

11. Gulcin I, Beydemir S, Hisar O. 2005. Effect of alpha-tocopherol on antioxidant enzyme activities and lipid peroxidation in rainbow trout Oncorhynchus mykiss. Acta Vet Hungarica 53: 425-433.
12. Gümüiş E, Ikiz R. 2009. Effect of dietary levels of lipid and carbohydrate on growth performance, chemical contents and digestibility in rainbow trout, Oncorhynchus mykiss Walbaum, 1792. Pakistan Vet J 29(2): 59-63.

13. Haghparast S, Kashiri H, Shabanpour B, Pahlavani MH. 2010. Antioxidant properties of sodium acetate, sodium citrate and sodium lactate on lipid oxidation in rainbow trout (Oncorhynchus mykiss) sticks during refrigerated storage $\left(4^{\circ} \mathrm{C}\right)$. Iran J Fisheries Sci 9(1): 73-86.

14. Hilton JW, Hodson PV, Slinger SJ. 1982. Absorption, distribution, half-life and possible routes of elimination of dietary selenium in juvenile rainbow trout (Salmo gairdneri). Comp Biochem Physiol C 71: 49-55.

15. Hopkins KD. 1992. Reporting fish growth: A review of the basics. J World Aquacult Soc 23: 173-179.

16. [ITP]. Instituto Tecnológico Pesquero del Perú. 2010. Determinación de Nitrógeno de Bases Volátiles Totales: Directiva 95/149. [Internet]. Disponible en: http://www.itp.gob.pe/

17. [ITP]. Instituto Tecnológico Pesquero del Perú. 2010. Determinación del ácido tiobarbiturico: Sinnhuber RO, Yu TC. 1977. TBA. [Internet]. Disponible en: http://www.itp.gob.pe/

18. Jasour MS, Rahimabadi EZ, Ehsani A, Rahnama M, Arshadi A. 2011. Effects of refrigerated storage on fillet lipid quality of rainbow trout (Oncorhynchus mykiss) supplemented by atocopheryl acetate through diet and direct addition after slaughtering. J Food Process Technol 2(5): 1-5.

19. Jittinandana $S$, Kenney PB, Slider SD, Kamireddy N, Hankins JS. 2006. High dietary vitamin $\mathrm{E}$ affects storage stability of frozen-refrigerated trout fillets. J Food Sci 71(2): C91-C96.

20. Kiaalvandi $S$, Faramarzi $M$, Iranshahi F, Jalaee MH. 2011. Effects of different fish meal as the main protein sources on the growth and 
feeding parameters of rainbow trout (Oncorhynchus mykiss). World J Fish Marine Sci 3(4): 265-268.

21. Küçükbay FZ, Yazlak H, Karaca I, Sahin N, Tuzcu M, Cakmak MN, Sahin K. 2009. The effects of dietary organic or inorganic selenium in rainbow trout (Oncorhynchus mykiss) under crowding conditions. Aquacult Nutr 15: 569-576.

22. Márquez F, Cabello AM, Villalobos LB, Guevara G, Figueroa BE, Vallenilla OM. 2006. Cambios físicos-químicos y microbiológicos observados durante el proceso tecnológico de la conserva de atún. Zootecnia Trop 24(1): 17-29.

23. Montaña C, Hurtado H, Gómez E. 2013. Rainbow trout (Oncorhynchus mykiss) fry initiation in closed water recirculation systems. Rev Colomb Cienc Pecu 26: 211-218.

24. Morales G. 2004. Crecimiento y eficiencia alimentaria de trucha arco iris (Oncorhynchus mykiss) en jaulas bajo diferentes regímenes de alimentación. Tesis de Ing. Agrónomo. Argentina: Universidad de Buenos Aires. 51 p.

25. Morris HJ, Carrillo O, Bermúdez RC. 2003. Enfoque integral en la utilización de los métodos químicos de evaluación de la calidad proteica. Rev Cub Salud Públ 29(1): 42-47.

26. [NTP] Norma Técnica Peruana. 2008. NTP 204.059. Trucha fresca. Método para medir el factor de conversión alimenticia. R.0067-2008. Lima: Indecopi.

27. Oldfield JE. 2003. Some recollections of early swine research with selenium and vitamin E. J Anim Sci 81(Suppl 2): E145-E148.

28. Oğuzhan P, Angiş S. 2012. Effect of salting and packaging treatments on fresh rainbow trout (Oncorhynchus mykiss) fillets during storage at refrigerator temperatures. Kafkas Univ Vet Fak Derg 18: 443-448.
29. Okumus I, Mazlum MD. 2002. Evaluation of commercial trout feeds: feed consumption, growth, feed conversion, carcass composition and bioeconomic Analysis. Turk J Fish Aquatic Sci 2: 101-107.

30. Özogul F, Özogul Y. 2000. Comparison of methods used for determination of total volatile basic nitrogen (TVB-N) in rainbow trout (Oncorhynchus mykiss). Turk J Zool 24: 113-120.

31. Packer L. 1991. Protective role of vitamin $\mathrm{E}$ in biological systems. Am J Clin Nutr 53: 1050S-1055S.

32. Palace VP, Spallholz JE, Holm J, Wautier K, Evans RE, Baron CL. 2004. Metabolism of selenomethionine by rainbow trout (Oncorhynchus mykiss) embryos can generate oxidative stress. Ecotox Environ Safe 58: 17-21.

33. Pearce J, Harris JE, Davies SJ. 2003. The effect of vitamin $E$ on the serum complement activity of the rainbow trout, Oncorhynchus mykiss (Walbaum). Aquacult Nutr 9: 337-340.

34. Pokniak J, De Bravó L, Galleguillos C, Battaglia J, Cornejo S. 1996. Respuesta productiva de la trucha arco iris (Oncorhynchus mykiss) a dietas extruidas con alto contenido lipídico. Av Cs Vet 11: 53-59.

35. Rider SA, Davies SJ, Jha AN, Clough $R$, Sweetman JW. 2010. Bioavailability of co-supplemented organic and inorganic zinc and selenium sources in a white fishmeal-based rainbow trout (Oncorhynchus mykiss) diet. J Anim Physiol Anim Nutr 94: 99-110.

36. Rowley AF, Knight J, Lloyd-Evans P, Holland JW, Vickers PJ. 1995. Eicosanoids and their role in immune modulation in fish-a brief overview. Fish Shellfish Immunol 5: 549-567.

37. Ruiz-Capillas C, Horner W. 1999. Determination of trimethylamine nitrogen and total volatile basic nitrogen in fresh fish by flow injection analysis. J Sci Food Agric 79: 1982-1986. 
38. Sara A, Ani AR, Barbu A. 2010. Influence of organic selenium on morphological characters and survival of fry and juvenile carp (Cyprinus carpio), Lausitz variety. Anim Sci Biotechnol 67: 326-333.

39. Sarmiento DF. 2011. Eficiencia productiva de trucha arco iris, bajo un sistema de recirculación de aguas con diferentes densidades de carga animal en la zona de Pailones, IASA, Ecuador. Tesis Ing Agropecuario. Ecuador: Escuela Politécnica del Ejército. $131 \mathrm{p}$.

40. [SAS]. Statistical Analysis System. 1995. User's guide.Cary, NC: SAS Institute Inc. USA. $163 \mathrm{p}$

41. Schrauzer GN. 2006. Selenium yeast: composition, quality, analysis, and safety. Pure Appl Chem 78: 105-109.

42. Sen CK, Khanna S, Roy S. 2006. Tocotrienols: vitamin E beyond tocopherols. Life Sci 78: 2088-2098.

43. Soetan KO, Olaiya CO, Oyewole OE. 2010. The importance of mineral elements for humans, domestic animals and plants: a review. Afr J Food Sci 4: 200-222.

44. Suárez H, Pardo SC, Cortés M, Ricaurte SC, Rojano B. 2009. Evaluación de nueva tecnología para mitigar las espinas intramus-culares en filetes de cachama Piaractus brachypomus
(Pisces: Characidae). Rev Fac Nac Agr Medellín 62(1): 4989-4997.

45. Trushenski JT, Kohler CC. 2007. Influence of stress and dietary naturalsource vitamin $\mathrm{E}$ on nonspecific immunocompetence, tissue tocopherol composition, and postslaughter fillet oxidative stability in sunshine bass. N Am J Aquacul 69: 330-339.

46. Van Dam B, Van Hinsbergh VW, Stehouwer CD, Versteilen A, Dekker $H$, Buytenhek R, et al. 2003. Vitamin $E$ inhibits lipid peroxidation-induced adhesion molecule expression in endothelial cells and decreases soluble cell adhesion molecules in healthy subjects. Cardiovas Res 57: 563-571.

47. Vinchira JE, Muñoz-Ramírez AP. 2010. Selenio: nutriente objetivo para mejorar la composición nutricional del pescado cultivado. Rev Med Vet Zoot 57: 59-75.

48. Yildiz M. 2004. The study of fillet quality and the growth performance of rainbow trout (Oncorhynchus mykiss) fed with diets containing different amounts of vitamin E. Turk J Fish Aquatic Sci 4: 81-86.

49. Zu K, Ip C. 2003. Synergy between selenium and vitamin $\mathrm{E}$ in apoptosis induction is associated with activation of distinctive initiator caspases in human prostate cancer cells. Cancer Res 63: 6988-6995. 